\title{
Avaliação histopatológica dos efeitos colaterais do uso intraestromal de biguanida em córnea de coelhos
}

\author{
Histopathological assessment of side effects \\ of the use of corneal intrastromal biguanide in rabbits
}

Lucas Vieira Bueno Rossi ${ }^{1}$, Eduardo Vieira Braga Espindola², Wilson Nahmatallah Obeid ${ }^{3}$, Fernando Buzatto Montovan ${ }^{4}$, Leonardo Guedes Marculino ${ }^{5}$, Vanessa Takayama ${ }^{6}$, Moacyr Pezati Rigueiro ${ }^{7}$, Denise de Freitas ${ }^{8}$, Luiz Antonio Vieira ${ }^{9}$

\section{Resumo}

Objetivo: Observar os efeitos da injeção intraestromal de biguanida a $0,02 \%, 0,1 \%$ e 0,5\% em córnea de coelhos. Métodos: Experimento prospectivo duplo cego. Foram utilizados 8 coelhos, identificados aleatoreamente e divididos em 4 grupos de 2 coelhos cada, ocorrendo a administração intraestromal de $0,2 \mathrm{ml}$ do fármaco na córnea do olho direito. O primeiro grupo recebeu biguanida na concentração de $0,02 \%$, o segundo grupo a $0,1 \%$, o terceiro a $0,5 \%$ e o quarto grupo (controle) recebeu a injeção de placebo - Soro Fisiológico $0,9 \%$. Um dos coelhos de cada grupo foi sacrificado no terceiro dia e o outro no décimo dia após a injeção da droga e tiveram os olhos direitos enucleados e submetidos a exame histopatológico. Resultados: Os coelhos que receberam placebo e biguanida a $0,02 \%$ apresentaram tanto no terceiro quanto no décimo dia da aplicação, reação inflamatória muito discreta. $\mathrm{O}$ coelho sacrificado no terceiro dia após a injeção da droga a $0,1 \%$ apresentou infiltrado inflamatório discreto com neutrófilos e eosinófilos, porém no décimo dia houve necrose estromal, vascularização e infiltrado intenso. O terceiro grupo apresentou necrose estromal, infiltrado moderado de leucócitos e atrofia endotelial com leucócitos na câmara anterior ao terceiro dia, evoluindo com necrose estromal extensa, infiltrado moderado e atrofia endotelial e epitelial no décimo dia. Conclusão: A biguanida quando utilizada via estromal nas concentrações de $0,1 \%$ e $0,5 \%$ pode causar efeitos indesejáveis na córnea de coelho, o que nos desperta para a necessidade de estudos posteriores com amostra maior para a confirmação dos achados.

Descritores: Coelhos; Córnea/efeitos de drogas; Toxicidade de drogas; Estroma da córnea; Avaliação de medicamentos/efeitos adversos

${ }^{1}$ Oftalmologista do Hospital CEMA - São Paulo (SP),Brasil;

${ }^{2}$ Oftalmologista do Hospital CEMA - São Paulo (SP), Brasil;

${ }^{3}$ Médico assistente do Setor de Córnea do Hospital CEMA - São Paulo (SP),Brasil;

${ }^{4}$ Oftalmologista do Hospital CEMA - São Paulo (SP), Brasil;

${ }^{5}$ Oftalmologista do Hospital CEMA - São Paulo (SP), Brasil;

${ }^{6}$ Oftalmologista do Hospital CEMA - São Paulo (SP), Brasil;

${ }^{7}$ Doutor, Chefe da disciplina de Patologia Cirúrgica do Departamento de Patologia da Universidade Federal de São Paulo - UNIFESP - São Paulo (SP), Brasil;

${ }^{8}$ Livre-docente, Professora Adjunta do Departamento de Oftalmologia da Universidade Federal de São Paulo - UNIFESP - São Paulo (SP), Brasil;

${ }^{9}$ Doutor, Chefe do Setor de Córnea do Hospital CEMA - São Paulo (SP), Brasil.

Trabalho realizado no Hospital CEMA. São Paulo (SP) - Brasil.

Recebido para publicação em: 22/2/2010 - Aceito para publicação em 28/8/2010 


\section{INTRODUÇÃO}

$\mathbf{O}$ grupo das biguanidas vem sendo estudado como potencial e versátil antimicrobiano, des de 1879, na preservação de cosméticos e produtos farmacêuticos principalmente como princípio ativo em formulações desinfetantes e sanitizantes para diversas áreas de aplicação ${ }^{(1-2)}$.

A biguanida polimérica, conhecida como PHMB Biguanida Polihexametileno -, foi sintetizada juntamente com a clorexidina nos laboratórios da ICI na Inglaterra ${ }^{(2)} \mathrm{e}$ denominada comercialmente como Vantocil IB. Estudos demonstram desempenho antimicrobiano superior da biguanida polimérica, quando comparada a outras biguanidas como, por exemplo, a clorexidina ${ }^{(7,3)}$. Por meio de pesquisas, constatou-se os benefícios da eficácia do PHMB como agente antimicrobiano ressaltados pelo amplo espectro de ação contra bactérias Gram-positivas, Gram-negativas, vírus e micro-organismos resistentes a antibióticos ${ }^{(2,3)}$.

Há décadas é estudado como ingrediente ativo em formulações desinfetantes, inclusive para uso em indústrias alimentícias, no controle de micro-organismos patogênicos como Escherichia coli, Staphylococcus aureus e Pseudomonas aeruginosa ${ }^{(2,3)}$.

Sua atividade é mantida na presença de matéria orgânica como leite, sangue ou albumina. Desinfetantes contendo PHMB como princípio ativo podem ser utilizados na desinfecção de equipamentos, pisos, paredes em sistemas abertos ou CIP (Clean-In-Place) ${ }^{(2,3)}$.

Em hospitais e áreas de saúde o PHMB também é indicado para desinfecção de pisos, paredes, mobiliários e artigos semicríticos. A sua eficácia no controle de bactérias resistentes a antibióticos e vírus pode ser um benefício extra para controle de infecções hospitalares ${ }^{(2,3)}$.

Em oftalmologia as biguanidas vêm sendo utilizadas no tratamento da ceratite por Acanthamoeba $a^{(4,10)}$. Também foi demonstrada a eficácia dessa droga no combate à proliferação de micro-organismos em recipientes de armazenamento de lentes de contato ${ }^{(11,12)}$, incluindo Pseudomonas $^{(13)}$ e Aspergillus $^{(14)}$. Outro uso na oftalmologia inclui utilização como antisséptico pré-operatório em cirurgias de catarata ${ }^{(15)}$.

O objetivo desse trabalho foi observar o efeito da injeção intraestromal de biguanida $0,02 \%, 0,1 \%$ e $0,5 \%$ em coelhos.

\section{MÉTOdos}

Foram utilizados neste experimento coelhos albinos da raça Nova Zelândia, 4 machos e 4 fêmeas, com peso vari- ando entre 2500 e $2800 \mathrm{~g}$, provenientes do Biotério da Faculdade de Ciências Médicas da Santa Casa de São Paulo. Os animais foram examinados pela médica veterinária responsável e mantidos na unidade de Técnica Cirúrgica e Cirurgia Experimental (UTECE / Santa Casa). O projeto de experimentação foi aprovado pela Comissão de Ética em Experimentação Animal (CEEA) desta mesma faculdade e o manuseio dos coelhos seguiu as normas e orientações do Comitê Brasileiro de Estudos em Animais de Experimentação (COBEA), da Association for Research in Vision and Ophthalmology (ARVO) e da UTECE/Santa Casa.

Os animais foram identificados aleatoriamente, marcados na face interna da orelha direita com caneta hidrográfica e divididos em 4 grupos de 2 coelhos cada, ocorrendo a administração de $0,2 \mathrm{ml}$ do fármaco em apenas um dos olhos (olho direito), por via intraestromal, em região temporal superior, após receberem anestesia apropriada. A dissecção das lamelas corneanas foi realizada com espátula crescente, com incisão corneana no limbo correspondente, formando-se assim um túnel estromal. Neste local foi aplicado o fármaco em estudo e a incisão corneana foi selada com hidratação estromal e com sutura corneana (01 ponto) com fio mononylon 10.0.

A droga apresentava a seguinte composição: (1) Polihexametil biguanida $(0,02,0,1$ ou $0,5 \%),(2)$ Soro Fisiológico 0,9\% (veículo de diluição).

O primeiro grupo recebeu biguanida na concentração de $0,02 \%$, o segundo grupo a $0,1 \%$, o terceiro a $0,5 \%$. O quarto grupo constituiu o grupo controle, recebendo a injeção de placebo - Soro Fisiológico 0,9\% (SF $0,9 \%)$. Um coelho de cada grupo foi sacrificado no $3^{\circ}$ dia após a injeção da droga e tiveram seus respectivos olhos direitos enucleados neste mesmo dia. Os demais coelhos foram sacrificados no $10^{\circ}$ dia, sendo igualmente submetidos à enucleação do olho direito. $\mathrm{O}$ procedimento de enucleação teve como prioridade a preservação da integridade da córnea $360^{\circ}$. Após tal procedimento os olhos foram imediatamente conservados em formol, tamponado a 10\% para subsequente exame histopatológico e avaliação dos efeitos causados pela droga.

Tanto a administração da droga quanto a enucleação dos coelhos foram realizadas na Unidade de Técnica Cirúrgica e Cirurgia Experimental da Faculdade de Ciências Médicas da Santa Casa de São Paulo entre os dias 07 e 17 de abril de 2008 (protocolo ${ }^{\circ} 160$ ).

\section{Resultados}

A avaliação histopatológica ocorreu em regime duplo-cego, com avaliação de fragmentos córneoesclerais 


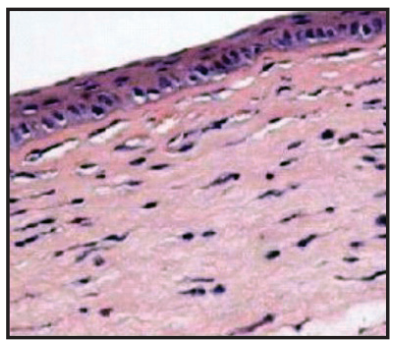

Figura 1: fotografia do corte histopatológico do terceiro dia após a injeção de biguanida a $0,02 \%$ (coelho do primeiro grupo). Processo inflamatório com neutrófilos e eosinófilos no estroma corneal. Tecido corado com hematoxilina-eosina. Aumento de $200 x$

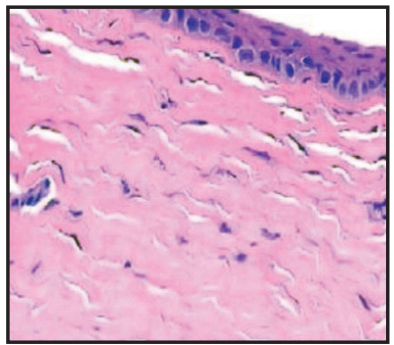

Figura 5: fotografia do corte histopatológico do décimo dia após a injeção de biguanida a $0,02 \%$ (coelho do primeiro grupo). Processo inflamatório com neutrófilos no estroma corneal. Tecido corado com hematoxilinaeosina. Aumento de 200x

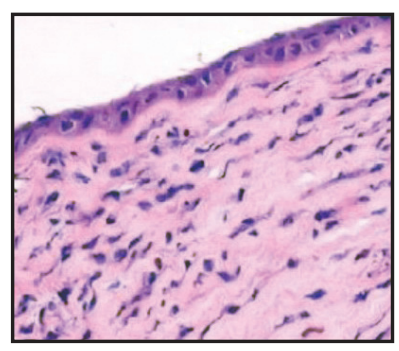

Figura 2: fotografia do corte histopatológico do terceiro dia após a injeção de biguanida a $0,1 \%$ (coelho do segundo grupo). Processo inflamatório com neutrófilos e eosinófilos no estroma corneal. Tecido corado com hematoxilina-eosina. Aumento de 200x

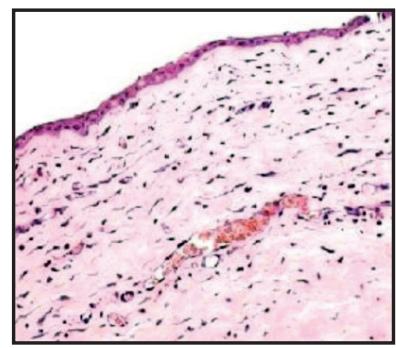

Figura 6: fotografia do corte histopatológico do décimo dia após a injeção de biguanida a $0,1 \%$ (coelho do segundo grupo). Presença de necrose e vascularização estromal. Tecido corado com hemotoxlinaeosina. Aumento de 200x

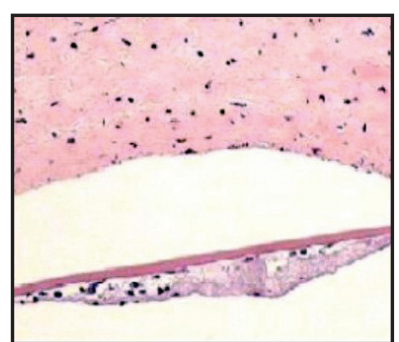

Figura 3: fotografia do corte histopatológico do terceiro dia após a injeção de biguanida a $0,5 \%$ (coelho do terceiro grupo). Presença de necrose e infiltrado de leucócitos no estroma corneal. Tecido corado com hematoxilina-eosina. Aumento de 200x

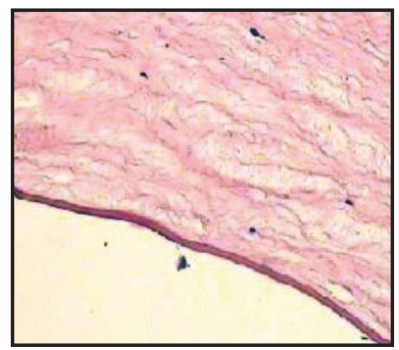

Figura 7: fotografia do corte histopatológico do décimo dia após a injeção de biguanida a $0,5 \%$ (coelho do terceiro grupo). Presença de necrose estromal, atrofia epitelial e endotelial. Tecido corado com hematoxilinaeosina. Aumento de 400x

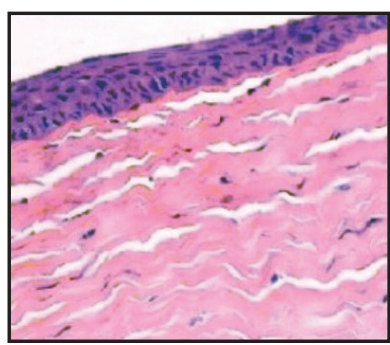

Figura 4: fotografia do corte histopatológico do terceiro dia após a injeção de SF 0,9\% (coelho do quarto grupo). Procesdo corado com hematoxilinaeosina. Aumento de 200x so inflamatório discreto. Teci-

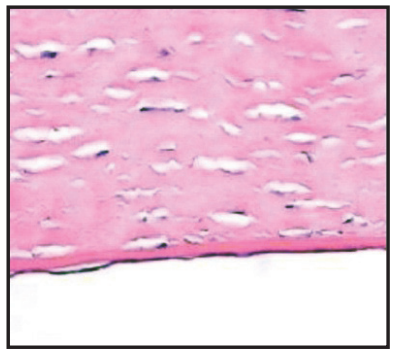

Figura 8: fotografia do corte histopatológico do décimo dia após a injeção de SF 0,9\% (coelho do quarto grupo). Processo inflamatório discreto. Tecido corado com hematoxilinaeosina. Aumento de 200x dos olhos dos 8 coelhos em estudo.

Dos 4 animais sacrificados, no terceiro dia após injeção da droga, 2 deles apresentaram infiltrado inflamatório discreto com neutrófilos e eosinófilos (Figuras 1 e 2) no local da injeção, respectivamente os que receberam biguanida a $0,02 \%$ (primeiro grupo) e biguanida a $0,1 \%$ (segundo grupo). No entanto, o coelho que recebeu a droga na concentração de $0,5 \%$ (terceiro grupo) apresentou ao exame histopatológico necrose estromal, infiltrado moderado de leucócitos e atrofia endotelial com leucócitos na câmara anterior (Figura 3). O coelho sacrificado que recebeu SF $0,9 \%$ (quarto grupo) apresentou inflamação muito discreta (Figura 4).

Os resultados do grupo sacrificado nesta primeira fase encontram-se na tabela 1.

Dentre os 4 coelhos restantes sacrificados no décimo dia, o que recebeu biguanida $0,02 \%$ (primei-

ro grupo) apresentou infiltrado inflamatório discreto com raros neutrófilos (Figura 5). No segundo grupo (biguanida a $0,1 \%$ ) observou-se necrose estromal, vascularização e infiltrado intenso (Figura 6). Já no terceiro grupo (biguanida a $0,5 \%$ ) ocorreu necrose extensa, infiltrado moderado e atrofia endotelial e epitelial (Figura 7). O coelho sacrificado que recebeu SF $0,9 \%$ (quarto grupo) apresentou inflamação muito discreta (Figura 8).

Os resultados do grupo sacrificado nesta segunda fase encontram-se na tabela 2.

\section{Discussão}

No presente estudo pode-se obter informações do aspecto histopatológico da reação da injeção de biguanida 
Tabela 1

\section{Resultado do estudo histopatológico das córneas examinadas ao terceiro dia após injeção de biguanida em diferentes concentrações}

\begin{tabular}{ll}
\hline $\begin{array}{l}\text { Concentração } \\
\text { de biguanida }\end{array}$ & Achados histopatológicos no $3^{\circ}$ dia \\
\hline $0 \%$ (placebo) & $\begin{array}{l}\text { Inflamação muito discreta } \\
\text { Infiltrado inflamatório discreto com } \\
\text { neutrófilos e eosinófilos }\end{array}$ \\
$0,02 \%$ & $\begin{array}{l}\text { Infiltrado inflamatório discreto com } \\
\text { neutrófilos e eosinófilos } \\
\text { Necrose estromal, infiltrado moderado } \\
\text { de leucócitos e atrofia endotelial com } \\
\text { leucócitos na câmara anterior. }\end{array}$ \\
\end{tabular}

no espaço intraestromal da córnea de coelhos. Quando administrada na concentração de $0,02 \%$ a substância provoca resposta inflamatória muito discreta, similar à da injeção de SF $0,9 \%$ neste mesmo local. Mesmo em um período de acompanhamento um pouco maior (10 dias) não se evidenciou resposta diferente em relação ao placebo, nem mudança significativa do padrão histopatológico em relação ao $3^{\circ}$ dia. No entanto, houve uma diferença significativa com o uso da biguanida a $0,1 \%$. Após 3 dias da aplicação da PHMB nesta concentração, evidenciou-se um infiltrado inflamatório discreto com neutrófilos e eosinófilos. No $10^{\circ}$ dia da injeção da mesma concentração o animal apresentou necrose estromal, vascularização e infiltrado intenso. Em contrapartida, quando se aumentou a concentração da droga para $0,5 \%$ foi possível observar uma reação diferente.Após 3 dias da aplicação da PHMB nesta concentração, evidenciou-se necrose estromal, infiltrado moderado de leucócitos e atrofia endotelial com leucócitos na câmara anterior. No $10^{\circ}$ dia da injeção da mesma concentração o animal apresentou necrose extensa, infiltrado moderado e atrofia endotelial e epitelial.

\section{ConClusão}

O estudo sugere, portanto, que a biguanida quando injetada no espaço intraestromal da córnea de coelhos nas concentrações de $0,02 \%$ comporta-se como o placebo (SF $0,9 \%$ ), não apresentando efeitos colaterais histopatológicos relevantes. No entanto, foram evidenciadas diversas alterações desencadeadas pela PHMB nas concentrações de $0,1 \%$ e $0,5 \%$, o que nos desperta para a necessidade de estudos posteriores com uma amostra maior para a confirmação dos achados.

\section{Tabela 2}

\section{Resultado do estudo histopatológico das córneas examinadas ao décimo dia após injeção de biguanida em diferentes concentrações}

\section{Concentração Achados histopatológicos no $10^{\circ}$ dia} de biguanida

$\begin{array}{ll}0 \% \text { (placebo) } & \text { Inflamação muito discreta } \\ 0,02 \% & \begin{array}{l}\text { Infiltrado inflamatório discreto com } \\ \text { raros neutrófilos }\end{array} \\ & \begin{array}{l}\text { Necrose estromal, vascularização } \\ \text { e infiltrado intenso }\end{array} \\ & \begin{array}{l}\text { Necrose extensa, infiltrado moderado } \\ \text { e atrofia endotelial e epitelial }\end{array} \\ \end{array}$

$0 \%$ (placebo) Inflamação muito discreta

\section{Abstract}

Purpose: Observe the effects of corneal intrastromal injection of biguanide at $0.02 \%, 0.1 \%$ and $0.5 \%$ in rabbits' eyes. Methods: Doble blind prospective study. 8 rabbits were used, randomly identified and divided into 4 groups, with 2 rabbits each, with the administration of $0.2 \mathrm{ml}$ of the drug via intrastromal, in the right eye. The first group received biguanide at a concentration of $0.02 \%$, the second one at $0.1 \%$, the third at $0.5 \%$ and the fourth group (control) was given a placebo injection - saline solution at $0.9 \%$. One rabbit from each group was sacrificed on the third day and the remaining rabbits on the tenth day after the drug injection and had its right eyes enucleated and submitted to histopathological analysis. Results: The rabbits that received placebo and biguanide at $0.02 \%$ presented, both on the third and the tenth day of the application, mild inflammation response. The rabbit sacrificed on the third day after the drug injection at $0.1 \%$ presented mild inflammatory infiltrate with neutrophils and eosinophils, although on the tenth day there was stromal necrosis, vascularization and intense infiltrate. The third group presented stromal necrosis, moderate infiltrate of leukocytes and endothelial atrophy with leukocytes in the anterior chamber on the third day, developing extensive stromal necrosis, moderate infiltrate and endothelial and epithelial atrophy on the tenth day. Conclusion: Biguanide when used at concentrations of $0.1 \%$ and $0.5 \%$ may cause adverse reactions in the rabbit's cornea, which arouses the necessity of further studies with a larger sample to confirm the findings.

Keywords: Rabbits; Cornea/drug effects; Drug toxicity; Corneal stroma; Drug evaluation/adverse effects 


\section{RefERÊNCIAS}

1. Katzung BG, editor. Farmacologia: básica e clínica. 9a ed. Rio de Janeiro: Guanabara Koogan; 2006. P. 1146.

2. Block SS, editor. Disinfection, sterilization and preservation. 5th ed. Philadelphia: Lippincott Williams \& Wilkins; 2001. p. 267-8.

3. Davies A, Field BS. The bactericidal action of biguanides. Biochem J. 1968;106(5):46.

4. Brasil. Ministério da Saúde. Agência Nacional de Vigilância Sanitária. Portaria ${ }^{\circ} 113$, de 22 de novembro de 1993. Portaria $\mathrm{n}^{\circ} 113$, de 22 de novembro de 1993. [citado 2007 mar 15]. Disponível em: http://www.anvisa.gov.br/legis/portarias/ 113_93.htm.

5. Nagington J, Richards JE. Chemotherapeutic compounds and Acanthamoebae from eye infections. J Clin Pathol. 1976;29(7):648-51.

6. Cohen EJ, Buchanan HW, Laughrea PA, Adams CP, Galentine PG, Visvesvara GS, et al. Diagnosis and management of Acanthamoeba keratitis. Am J Ophthalmol. 1985;100(3):389-95.

7. Stehr-Green JK, Bailey TM, Visvesvara GS. The epidemiology of Acanthamoeba keratitis in the United States. Am J Ophthalmol. 1989;107(4):331-6.

8. Obeid WN, Araújo R, Vieira LA, Machado MAC. Ceratite bilateral por Acanthamoeba: relato de caso. Arqq Bras Oftalmol. 2003;66(6):876-80.

9. Larkin DF, Kilvington S, Dart JK. Treatment of Acanthamoeba keratitis with polyhexamethylene biguanide. Ophthalmology. 1992;99(2):185-91.

10. Donoso Rojas R, Mura C JJ, Lopez M M. Queratitis por Acanthamoeba tratadas con propamidina y polihexametil biguanida: PHMB. Rev Med Chile. 2002;130(4):396-401.
11. Rosenthal RA, Dassanayake NL, Schlitzer RL, Schlech BA, Meadows DL, Stone RP. Biocide uptake in contact lenses and loss of fungicidal activity during storage of contact lenses. Eye Contact Lens. 2006;32(6):262-6.

12. Hughes R, Heaselgrave W, Kilvington S. Acanthamoeba polyphaga strain age and method of cyst production influence the observed efficacy of therapeutic agents and contact lens disinfectants. Antimicrob Agents Chemother. 2003;47(10):3080-4.

13. Codling CE, Maillard JY, Russell AD. Performance of contact lens disinfecting solutions against Pseudomonas aeruginosa in the presence of organic load. Eye Contact Lens. 2003;29(2):100-2.

14. Panda A, Ahuja R, Biswas NR, Satpathy G, Khokhar S. Role of $0.02 \%$ polyhexamethylene biguanide and $1 \%$ povidone iodine in experimental Aspergillus keratitis. Cornea. 2004;22(2):138-41.

15. Hansmann F, Kramer A, Ohgke H, Strobel H, Müller M, Geerling G. [Polyhexamethylbiguanid (PHMB) as preoperative antiseptic for cataract surgery]. Ophthalmologe. 2004;101(4):377-83. German.
Endereço para correspondência:
Lucas Vieira Bueno Rossi
Rua Campo Largo ${ }^{\circ} 252$ - apto 11 - Mooca
CEP 03186-010 - São Paulo (SP), Brasil
Tel: 11-26046962 / Fax: 19-33074551
lucasvbrossi@msn.com 